\title{
Discovery of near-ultraviolet counterparts to millisecond pulsars in the globular cluster 47 Tucanae $^{\star}$
}

L. E. Rivera-Sandoval, ${ }^{1} \dagger$ M. van den Berg, ${ }^{1,2}$ C. O. Heinke, ${ }^{3,4}$ H. N. Cohn, ${ }^{5}$ P. M. Lugger, ${ }^{5}$ P. Freire, ${ }^{4}$ J. Anderson, ${ }^{6}$ A. M. Serenelli, ${ }^{7}$ L. G. Althaus, ${ }^{8}$ A. M. Cool,${ }^{9}$ J. E. Grindlay, ${ }^{2}$ P. D. Edmonds, ${ }^{2}$ R. Wijnands ${ }^{1}$ and N. Ivanova ${ }^{3}$

${ }^{1}$ Anton Pannekoek Institute for Astronomy, University of Amsterdam, Science Park 904, NL-1098 XH Amsterdam, the Netherlands

${ }^{2}$ Harvard-Smithsonian Center for Astrophysics, 60 Garden Street, Cambridge, MA 02138, USA

${ }^{3}$ Department of Physics, University of Alberta, CCIS 4-183, Edmonton, AB T6G 2E1, Canada

${ }^{4}$ Max Planck Institute for Radio Astronomy, Auf dem Hugel 69, D-53121 Bonn, Germany

${ }^{5}$ Department of Astronomy, Indiana University, 727 E. Third St, Bloomington, IN 47405, USA

${ }^{6}$ Space Telescope Science Institute, 3700 San Martin Drive, Baltimore, MD 21218, USA

${ }^{7}$ Instituto de Ciencias del Espacio (CSIC-IEEC), Facultad de Ciencias, Campus UAB, E-08193 Bellaterra, Spain

${ }^{8}$ Instituto de Astrofísica La Plata, Facultad de Ciencias Astronómicas y Geofísicas (CONICET-UNLP) Paseo del Bosque s/n, 1900 La Plata, Argentina

${ }^{9}$ Department of Physics and Astronomy, San Francisco State University, 1600 Holloway Avenue, San Francisco, CA 94132, USA

Accepted 2015 August 4. Received 2015 August 1; in original form 2015 June 26

\begin{abstract}
We report the discovery of the likely white dwarf companions to radio millisecond pulsars 47 Tuc Q and 47 Tuc $\mathrm{S}$ in the globular cluster 47 Tucanae. These blue stars were found in near-ultraviolet images from the Hubble Space Telescope for which we derived accurate absolute astrometry, and are located at positions consistent with the radio coordinates to within $0.016 \operatorname{arcsec}(0.2 \sigma)$. We present near-ultraviolet and optical colours for the previously identified companion to millisecond pulsar 47 Tuc $\mathrm{U}$, and we unambiguously confirm the tentative prior identifications of the optical counterparts to 47 Tuc T and 47 Tuc Y. For the latter, we present its radio-timing solution for the first time. We find that all five near-ultraviolet counterparts have $U_{300}-B_{390}$ colours that are consistent with He white dwarf cooling models for masses $\sim 0.16-0.3 \mathrm{M} \odot$ and cooling ages within $\sim 0.1-6$ Gyr. The $\mathrm{H} \alpha-R_{625}$ colours of 47 Tuc $\mathrm{U}$ and 47 Tuc $\mathrm{T}$ indicate the presence of a strong $\mathrm{H} \alpha$ absorption line, as expected for white dwarfs with an $\mathrm{H}$ envelope.
\end{abstract}

Key words: pulsars: individual: PSR J0024-7204Q-pulsars: individual: PSR J00247204S - pulsars: individual: PSR J0024-7204T - pulsars: individual: PSR J0024-7203U pulsars: individual: PSR J0024-7204Y - globular clusters: individual: 47 Tucanae.

\section{INTRODUCTION}

Millisecond pulsars (MSPs) are rapidly spinning neutron stars with spin periods of $20 \mathrm{~ms}$ or less, magnetic field strengths $B \approx 10^{8-9} \mathrm{G}$, and characteristic spin-down ages $\tau_{\mathrm{c}} \approx 10^{9-10} \mathrm{yr}$ (see e.g. the review by Ransom 2008). Their properties can be explained by the recycling formation scenario (Alpar et al. 1982), in which an old neutron star is spun up by the accretion of mass from a companion star in a low-mass X-ray binary (LMXB) phase.

\footnotetext{
${ }^{\star}$ Based on proprietary and archival observations with the NASA/ESA Hubble Space Telescope, obtained at the Space Telescope Science Institute, which is operated by AURA, Inc., under NASA contract NAS 5-26555.

†E-mail: 1.e.riverasandoval@uva.nl
}

Up to now, more than 200 MSPs have been discovered in the Galaxy, and a large fraction of these reside in globular clusters (GCs). The number of MSPs per unit mass is higher in GCs than in the Galactic disc by two to three orders of magnitude (Freire 2013). In the context of the recycling scenario, this overabundance is no surprise. The LMXB progenitors of MSPs have long been known to occur in GCs with a much higher frequency than in the rest of the Galaxy (Clark 1975) as a result of the high stellar encounter rates that favour their formation in dense clusters.

Most MSPs are found in binary systems. Studies of the orbital parameters and the companion properties can provide valuable information on the advanced evolution of LMXBs, the pulsar spin-up process (e.g. Smarr \& Blandford 1976), and (for MSPs in GCs) the effects of dynamical encounters on the characteristics of the MSP population (see e.g. Verbunt \& Freire 2014). Various classes of binary MSPs can be distinguished; each of these is found in the 
Galactic field and in GCs. The systems that are expected to result from the standard recycling scenario have low-mass (a few tenths of $\mathrm{M}_{\odot}$ ) white dwarf (WD) companions that descended from the original mass donors in the LMXBs. Most MSPs are found in this configuration. The very low mass binary MSPs (or black widows) likely represent the very latest stages of evolution, in which the companion (with only a few hundredths of $\mathrm{M}_{\odot}$ in mass remaining) is being ablated by the pulsar. Finally, the so-called redback MSPs are characterized by radio eclipses and non-degenerate companions of $0.2-0.4 \mathrm{M}_{\odot}$ (see Roberts 2013 for a review). These may be systems in transition between an LMXB phase and a detached phase where the MSP can be detected as a radio pulsar or (in GCs) the result of exchange encounters.

The identification of MSPs at other wavelengths enables a range of studies into the physical processes that govern the evolution of the MSP binaries. In GCs, the spatial resolution of the Chandra X-ray Observatory and the Hubble Space Telescope (HST) is a necessity to overcome the crowding. Indeed, the first unambiguous X-ray identification of MSPs in GCs was made with the first Chandra observation of 47 Tuc, in which 15 of the 23 MSPs then known were first detected and precisely located in X-rays (Grindlay et al. 2001). So far, HST counterparts to GC MSPs have been found for only 12 systems, 2 of which are tentative identifications (47 Tuc $\mathrm{T}$ and Y; Edmonds et al. 2003a). The other 10 systems in GCs are 47 Tuc U and W (Edmonds et al. 2001, 2002), NGC 6397 A (Ferraro et al. 2001), NGC 6752 A (Bassa et al. 2003; Ferraro et al. 2003), M4 A (Sigurdsson et al. 2003), NGC 6266 B (Cocozza et al. 2008), M28 H and I (Pallanca et al. 2010, 2013), M5 C (Pallanca et al. 2014), and M71 A (Cadelano et al. 2015). Clearly, more identifications are needed to get a better understanding of the possible differences between MSP properties in GCs and in the field.

Right after Terzan 5, 47 Tucanae (47 Tuc) is the GC where most radio MSPs have been identified so far: a total of 23 were discovered (Manchester et al. 1990, 1991; Robinson et al. 1995; Camilo et al. 2000), 15 of which are in binary systems. ${ }^{1}$ To date, accurate radiotiming positions have been derived for 13 of the 47 Tuc binary MSPs (Freire et al. 2003, and in preparation), which allow a search for their counterparts at ultraviolet and optical wavelengths. So far, secure identifications have been found for only two of them. The companion of PSR J0024-7204W, or 47 Tuc W in short, is a non-degenerate star that may be (close to) filling its Roche lobe (Edmonds et al. 2002; Bogdanov, Grindlay \& van den Berg 2005); it was among the first MSP binaries to be classified as a 'redback' system. The companion of 47 Tuc U, on the other hand, is typical for a system that is the outcome of the canonical recycling scenario. Edmonds et al. (2001, E01 hereafter) found that it is likely an $\mathrm{He}$ $\mathrm{WD}^{2}$ with a mass of $\sim 0.17 \mathrm{M}_{\odot}$. Edmonds et al. (2003a, from now on E03a) suggested identifications for two more systems, 47 Tuc $\mathrm{Y}^{3}$ and $\mathrm{T}$, but from their data they could only derive upper limits on the $U-V$ colours of the proposed blue counterparts.

\footnotetext{
${ }^{1}$ http://www.naic.edu/ pfreire/GCpsr.html

${ }^{2} \mathrm{He}$ WDs are low-mass $\left(\lesssim 0.45 \mathrm{M}_{\odot}\right)$ WDs with He-rich cores. They are thought to have formed as a result of a mass-transfer episode in a close binary before He ignition, removing much of the He WD progenitor's envelope and exposing its $\mathrm{He}$-rich core.

${ }^{3}$ The optical counterpart to 47 Tuc Y was originally reported as the counterpart to the Chandra source W 82 by E03a. This source was classified as a candidate cataclysmic variable, as the radio position for 47 Tuc $\mathrm{Y}$ was unknown at the time. After the position became available, Bogdanov et al. (2006) reported its association with W 82, implying the detection of the MSP companion by E03a.
}

We have carried out a search for counterparts to the 47 Tuc MSPs using new near-ultraviolet (NUV) imaging data obtained with the HST Wide Field Camera 3 (WFC3) and optical data (including deep $\mathrm{H} \alpha$ imaging) from the Advanced Camera for Surveys (ACS). The NUV data are particularly useful for looking for WD companions, whose blue spectral energy distribution yields a high contrast against the typically red cluster stars. Here we present the results of our search, which includes the discovery of the counterparts to 47 Tuc $\mathrm{Q}$ and $\mathrm{S}$, and the corroboration of the suggested identifications of 47 Tuc T and Y. We find that, like in 47 Tuc U, the MSP companion in all four systems is likely a low-mass $\left(0.16-0.3 \mathrm{M}_{\odot}\right) \mathrm{He}$ WD.

This paper is structured as follows. In Section 2, we describe the HST observations and the X-ray data used in this work. The HST data analysis is described in Section 3. In Section 4, we present the search for counterparts to the 47 Tuc MSPs in the HST data, and we show our results for the new identifications and the known WD companion of 47 Tuc U. Finally, we discuss our results in Section 5.

\section{OBSERVATIONS}

The NUV data used in this work ${ }^{4}$ belong to HST programme GO 12950 taken with the UVIS channel of the WFC3. The data set consists of eight orbits divided into two consecutive visits on 2013 August 13 starting at 02:10 UTC and finishing at 11:01 UTC of the same day. Each visit started with one orbit of four F390W exposures of about $580 \mathrm{~s}$ each. This was followed by three orbits of 12 F300X exposures in total, with individual exposure times of about $609 \mathrm{~s}$, resulting in a total exposure time of $3881 \mathrm{~s}$ in the F390W filter and $14256 \mathrm{~s}$ in the F300X filter. The native scale of the WFC3/UVIS images is 0.04 arcsec pixel $^{-1}$. The UVIS detector is composed of two chips, having a chip gap between them equivalent to $1.2 \operatorname{arcsec}$ on the sky. In order to improve our spatial resolution, we used a fourpoint dithering pattern that includes fractional-pixel offsets. In both visits, an offset between images was imposed to ensure coverage of the chip gap. The images were centred near the centre of 47 Tuc as reported by Goldsbury et al. (2011). Given that the WFC3/UVIS has a field of view (FOV) of $162 \operatorname{arcsec} \times 162$ arcsec, our images only cover $\sim 23$ percent of the area inside the half-light radius of the cluster $\left.\left[r_{\mathrm{h}}=3.17 \text { arcmin; Harris } 1996 \text { (2010 edition }\right)^{5}\right]$.

The optical observations reported here were taken with the Wide Field Channel (WFC) on the ACS under programme GO 9281. We obtained images in three filters: F435W, F658N, and F625W, which encompass $\mathrm{H} \alpha$ and the surrounding continuum. The observations were spread over three visits on 2002 September 30 (01:25-06:31 UTC), October 2/3 (20:37-00:51 UTC), and October 11 (02:0410:40 UTC). The visits were scheduled to be simultaneous with the deep 47 Tuc Chandra observations that are presented in Heinke et al. (2005, hereafter H05). The total exposure time amounted to $955 \mathrm{~s}$ in F435W, $1320 \mathrm{~s}$ in F625W, and $7440 \mathrm{~s}$ in F685N. Observations through all three filters were taken in each visit, resulting in near-simultaneous colours. Since X-ray emitting binaries frequently display variability in X-ray and optical wavelengths, this provides an optimal estimate of the system's actual colours, which is unaffected by long-term variability in one or both wavelength bands.

\footnotetext{
${ }^{4}$ We have not analysed the existing Space Telescope Imaging Spectrograph (STIS) far-ultraviolet data of the centre of 47 Tuc, because the five objects studied in this paper are not included in the small field of view of that data set.

${ }^{5}$ Catalogue of parameters for Milky Way GCs, http://www.physics. mcmaster.ca/Fac_Harris/mwgc.dat
} 
The FOV of the WFC is $202 \operatorname{arcsec} \times 202$ arcsec, imaged with two CCD detectors separated by a 2.45 -arcsec-wide gap. We used a dither pattern with fractional-pixel offsets to be able to improve the native WFC pixel scale of 0.05 arcsec pixel ${ }^{-1}$ during the image stacking. A complete description of the GO 9281 data and results is forthcoming (van den Berg et al., in preparation). Of the 20 MSPs in 47 Tuc with radio-timing positions, 19 are covered by the UVIS and WFC images; only 47 Tuc X, a binary MSP, was missed.

In this work, we make use of the catalogue of X-ray sources in 47 Tuc from $\mathrm{H} 05$ (see that paper for details about the data reduction), which was obtained from deep observations (281 ks in total) with Chandra. All 19 MSPs covered by our NUV/optical data are matched to an X-ray source in this catalogue.

\section{DATA REDUCTION AND ANALYSIS}

The data reduction of our UVIS data starts with the flat-fielded images produced by the standard UVIS pipeline (CALWF3 version 3.1.2). To correct for the charge transfer efficiency (CTE) degradation of the UVIS detectors, we used the CTE correction software ${ }^{6}$ provided by the Space Telescope Science Institute (STScI). The resulting images still suffer from geometric distortion, which we corrected for with the DRIZZLEPAC ${ }^{7}$ software (Gonzaga et al. 2012). Astrometric alignment of the individual images was performed with the TWEAKREG task in DRIZZLEPAC. Subsequently, the ASTRODRIZZLE task was used to combine the aligned images into one stacked master frame in each filter that is distortion free, and cleaned of cosmic rays and detector artefacts like hot pixels or bad columns. The resulting master images in F390W and F300X are twice-oversampled to a pixel scale of 0.02 arcsec pixel ${ }^{-1}$.

The reduction steps for the WFC images are similar to those for the UVIS data, except that the images produced by the ACS calibration pipeline (CALACS version 8.2.0) are already corrected for CTE losses. The stacked master images in F435W, F658N, and F625W have a final pixel scale of $0.025 \operatorname{arcsec}_{\text {pixel }}{ }^{-1}$.

\subsection{Astrometry}

The radio coordinates of the 47 Tuc MSPs were derived using the Solar system ephemeris DE/LE 405. This is specified in the International Celestial Reference Frame, which is aligned to the International Celestial Reference System (ICRS). To facilitate the identification of NUV/optical counterparts, we also aligned our UVIS and WFC images to the ICRS using stars in the UCAC2 catalogue (Zacharias et al. 2004). Since isolated and unsaturated UCAC2 stars are scarce in the HST images of the cluster core, we derived secondary astrometric standards from a ground-based image of 47 Tuc as an intermediate step. We obtained from the ESO archive a $30 \mathrm{~s} V$ image taken with the $2.2 \mathrm{~m} /$ Wide Field Imager (WFI) at La Silla, Chile on 2002 October 29. An astrometric solution of the part of the image that contains the cluster centre was derived using 225 UCAC2 stars; fitting for zero-point offset, rotation, pixel scale, and distortions gives rms residuals of about 0.035 arcsec in right ascension and declination. We then selected 126 secondary standards from the WFI image to calibrate a $10 \mathrm{~s} \mathrm{~F} 435 \mathrm{~W}$ exposure from our GO 9281 programme, which resulted in rms residuals of about 0.027 arcsec in each direction. Finally, this solution was transferred to our stacked WFC images with negligible errors using

\footnotetext{
${ }^{6}$ http://www.stsci.edu/hst/wfc3/tools/cte_tools

${ }^{7}$ http://drizzlepac.stsci.edu
}

$>10000$ stars, and to our stacked UVIS images with rms residuals of about 0.027 arcsec in each direction using $>11700$ stars. The final $1 \sigma$ error in the absolute astrometry of our images is the quadratic sum of all the aforementioned errors, plus a term that represents the error with which the UCAC2 is tied to the ICRS. The latter is dominated by the estimated systematic error in UCAC2 positions of 10 mas (Zacharias et al. 2004). For the WFC and UVIS images, this results in an error of 0.064 and 0.074 arcsec, respectively. Errors in the radio positions are negligible.

H05 aligned the astrometry of their X-ray source catalogue to the radio positions of 17 MSPs detected by Chandra. Therefore, we can indirectly check the alignment between our NUV and radio positions by comparing the NUV and Chandra astrometry. We have done this by comparing the NUV and X-ray positions of 17 sources with published finding charts in E03a and Edmonds et al. (2003b). We find that the NUV/X-ray offset is very small, about $-0^{\prime \prime} 023 \pm 00^{\prime \prime} 008$ in right ascension and $-0.003 \pm 00^{\prime \prime} 009$ in declination in both filters, so about one oversampled pixel.

\subsection{Photometry}

Given that the images of the core of 47 Tuc are very crowded, we carried out point spread function (PSF) photometry for the stacked UVIS and WFC frames by using standard IRAF and DAOPHOT tasks. We used isolated stars to build a variable PSF for each filter. The obtained PSF was fitted to all detected stars in each stacked frame. Photometric calibration of the extracted magnitudes was performed on to the Vega-mag system using the zero-points given at STScI's web pages $;{ }^{8}$ for UVIS we used the zero-points provided for 0.4 arcsec apertures. In the remainder, we denote calibrated magnitudes in the F300X, F390W, F435W, F625W, and F658N filters by $U_{300}, B_{390}, B_{435}, R_{625}$, and $\mathrm{H} \alpha$, respectively. The absolute magnitudes were calculated for an adopted distance modulus $(m-$ $M)_{0}=13.36 \pm 0.02 \pm 0.06$, corresponding to the 47 Tuc distance of $4.69 \pm 0.04 \pm 0.13 \mathrm{kpc}$ (Woodley et al. 2012). The first error term corresponds to the random error, and the second to the systematic error of the distance determination.

The UVIS data were also independently analysed using software based on the program developed for the ACS Globular Cluster Treasury Project, described in Anderson et al. (2008), which gave consistent results with the DAOPHOT analysis. The reductions were done using the latest version of this software known as Ks2. Star finding required that stars be detected in both the F300X and F390W frames. Photometry was performed by PSF fitting using library PSFs with perturbations to better match the specific PSF in each exposure. The final Ks 2 photometry comes from averaging the fluxes from the individual images, with sigma clipping applied to remove outlying values due to cosmic rays, defective pixels, etc. A total of 148717 stars were detected in the region covered by the UVIS imaging. Colour-magnitude diagrams (CMDs) were constructed to choose the best $\mathrm{Ks} 2$ options for photometry of the faint stars (e.g. the fitting radius), from the tightness of the fiducial sequences, and to identify the counterparts for the MSPs. DRIZZLE-combined mosaic images of this region were also produced using the STScI PYRAF routine ASTRODRIZZLE from the DRIZZLEPAC package. The DRIZZLE-combined images were oversampled by a factor of 2 , in order to increase the effective resolution. Calibration of the $\mathrm{ks} 2$ photometry to the Vega-mag system was performed by doing aperture photometry on moderately bright, isolated stars in the image

\footnotetext{
${ }^{8}$ http://www.stsci.edu/hst/wfc3/phot_zp_lbn
}

http://www.stsci.edu/hst/acs/analysis/zeropoints 
mosaics within a 0.1 arcsec radius aperture, finding the aperture correction to an infinite-radius aperture from ISR WFC3 2009-38, calculating the median offset between the Ks 2 photometry and the aperture photometry for these stars, and applying the zero-points from the HST WFC3 zero-point website. ${ }^{9}$

To take into account the effect of extinction on the UVIS photometry, we converted the reddening towards 47 Tuc of $E(B-V)=0.04 \pm 0.02$ (Salaris et al. 2007) to the extinction in the F300X and F390W filters using the UVIS Exposure Time Calculator of $H S T$. We obtained extinction values of $A(\mathrm{~F} 300 \mathrm{X})=0.26 \pm$ 0.13 and $A(\mathrm{~F} 390 \mathrm{~W})=0.18 \pm 0.09$, respectively. To compute the extinction in the WFC filters, we used the conversions in Sirianni et al. (2005). This yields $A(\mathrm{~F} 435 \mathrm{~W})=0.16 \pm 0.08, A(F 658 N)=0.10 \pm$ 0.05 , and $A(\mathrm{~F} 625 \mathrm{~W})=0.11 \pm 0.05$.

\section{RESULTS}

The search for MSP counterparts was done in the astrometrically calibrated NUV images. These frames are less crowded than the optical images, and contain fewer bright and saturated stars that could affect the detection of faint counterparts. We looked for counterparts within $3 \sigma(0.22 \mathrm{arcsec})$ of the radio positions.

We found blue stars inside the match circles of the binary MSPs 47 Tuc Q, S, T, U, and Y. These objects are excellent astrometric matches with the radio positions, with offsets that are smaller than $0.016 \operatorname{arcsec}(0.2 \sigma)$. All other stars inside the $3 \sigma$ error circles lie further from the radio positions, and do not have blue $U_{300}-B_{390}$ colours.

The blue stars that match with 47 Tuc T, Y and U are the likely WD counterparts proposed by E01, E03a, and Bogdanov et al. (2006), while for 47 Tuc Q and S these are new discoveries. Based on the similar locations in the $B_{390}$ versus $U_{300}-B_{390} \mathrm{CMD}$, and the good alignment with the radio positions, we consider the stars near 47 Tuc $\mathrm{Q}$ and $\mathrm{S}$ to be the likely counterparts, and WD companions, to these MSPs, as well. A more detailed discussion of the individual systems is given in Sections 4.1-4.5. In the remainder of the text, we use capital letters (without subscript) to refer to the MSPs detected at radio wavelengths, while we add the subscript ' $U V$ ' to refer to the NUV counterparts, e.g. 47 Tuc Q versus Quv. Finding charts in the F300X and F390W filters are presented in Fig. 1. Figs 2 and 3 are NUV and optical CMDs for 47 Tuc that show the photometry for $\mathrm{Q}_{\mathrm{UV}}, \mathrm{S}_{\mathrm{UV}}, \mathrm{T}_{\mathrm{UV}}, \mathrm{U}_{\mathrm{UV}}$, and $\mathrm{Y}_{\mathrm{UV}}$. In Table 1, we summarize the properties of the five MSPs as determined from radio and X-ray observations, while our astrometric and photometric results can be found in Table 2.

We have estimated the parameters of $\mathrm{Q}_{\mathrm{UV}}, \mathrm{S}_{\mathrm{UV}}, \mathrm{T}_{\mathrm{UV}}, \mathrm{U}_{\mathrm{UV}}$, and $\mathrm{Y}_{\mathrm{UV}}$ assuming that they are He WDs. In Figs 2 and 3, we have included new evolutionary tracks for He WD cooling models as described in Serenelli et al. (2002), but with a metallicity as appropriate for 47 Tuc of $Z=0.003([\mathrm{Fe} / \mathrm{H}]=-0.78$; Thygesen et al. 2014). Masses were estimated by determining the mass range that corresponds to the two cooling tracks that bracket the location of the companion in the NUV CMD, including $1 \sigma$ error bars. Errors include the DАОРНОТ uncertainties, errors in the calibration, and in the adopted distance modulus and extinction (see Section 3.2). The corresponding ranges in age, effective temperature $T_{\text {eff }}$, surface gravity $\log g$, and bolometric luminosity $L$ were determined by interpolating linearly along the two bracketing tracks using the

${ }^{9}$ http://www.stsci.edu/hst/wfc3/documents/ISRs/WFC3-2009-38.pdf
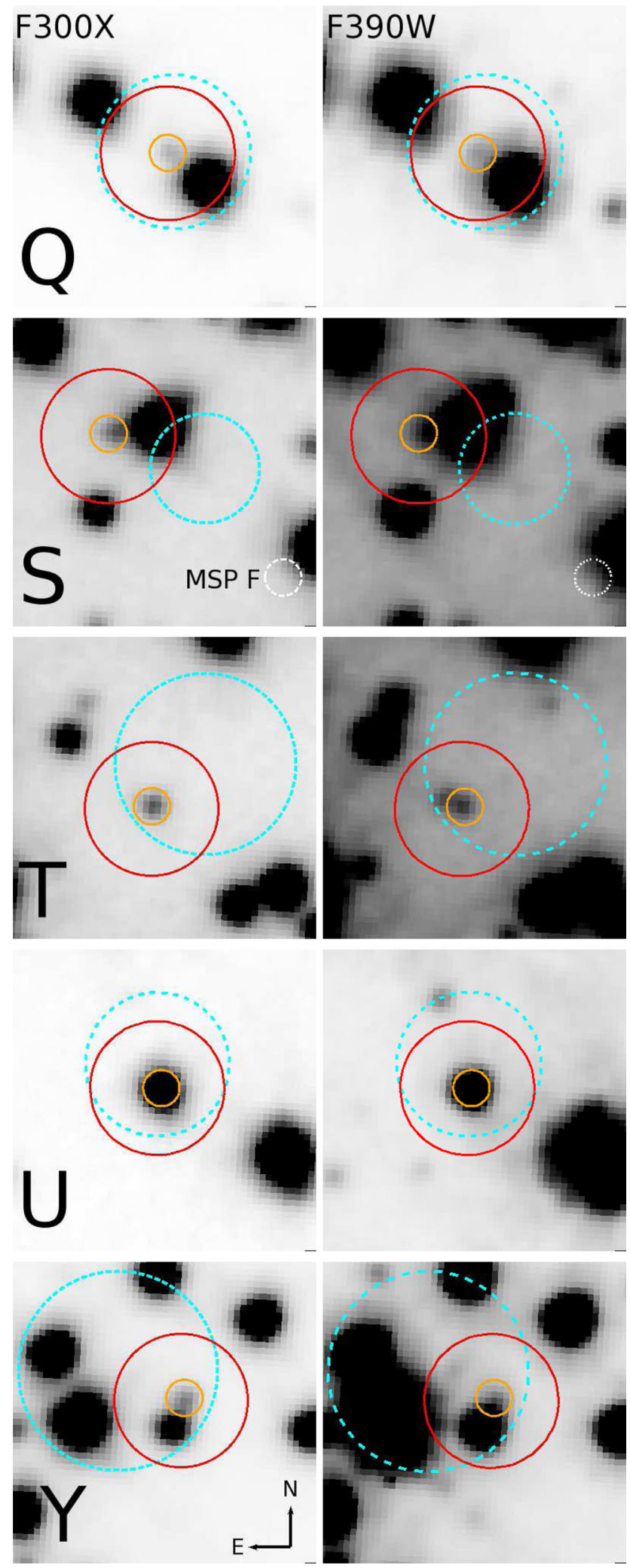

Figure 1. Finding charts of the companions to MSPs 47 Tuc Q, S, T, U, and Y. Images in the filter F300X are shown on the left side while images in F390W are shown on the right. The solid red circles show the $3 \sigma$ match circles centred on radio positions of the pulsars, which take into account the astrometric UVIS errors. The dashed cyan circles are the $3 \sigma$ match circles of Chandra sources. The companion candidates are indicated by solid orange circles. Each image is $1.0 \operatorname{arcsec} \times 1.0 \operatorname{arcsec}$ in size. 


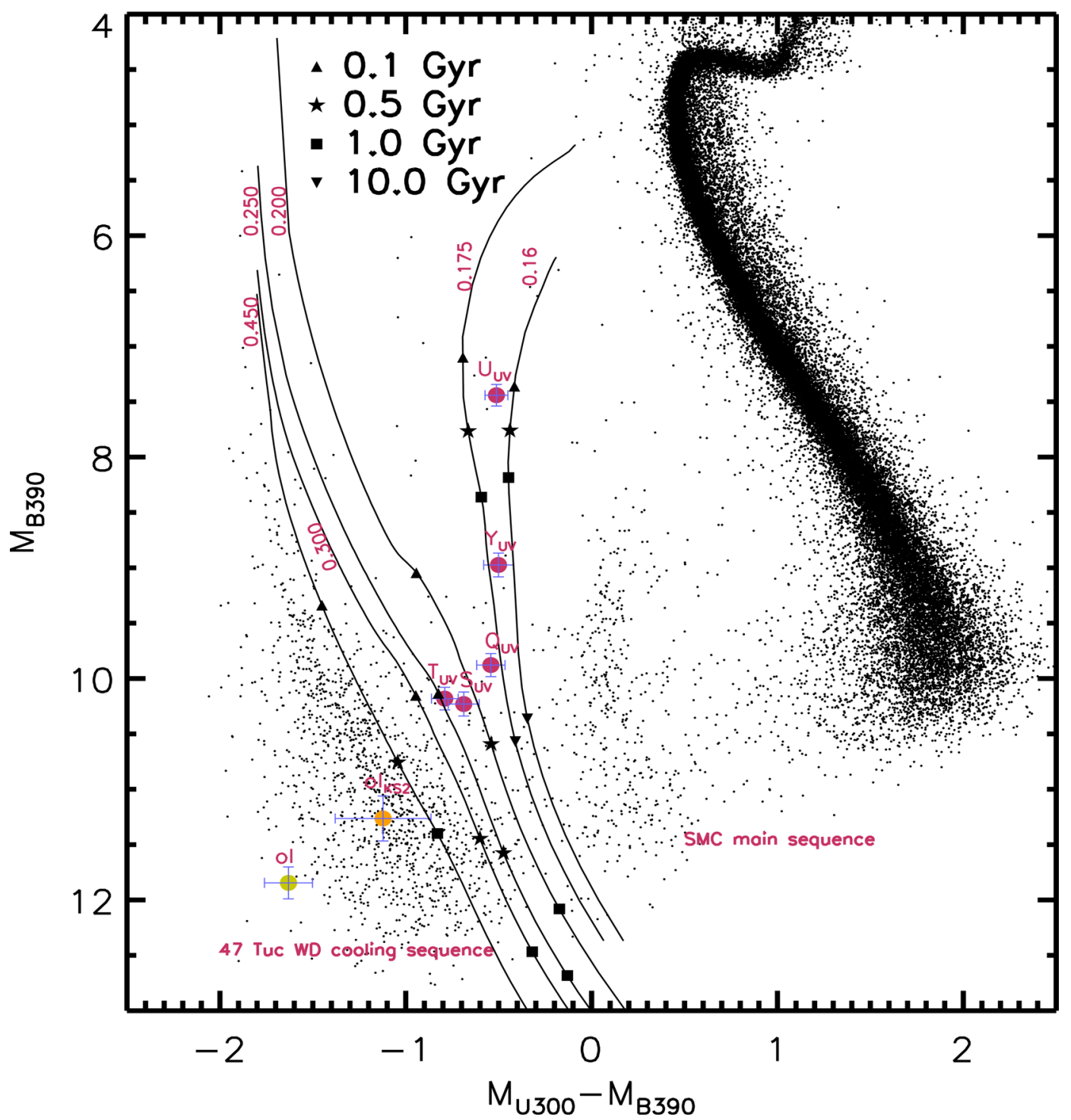

Figure 2. $U_{300}-B_{390}$ versus $B_{390}$ CMD based on DAорнот photometry of our UVIS images. Each MSP companion is denoted with a letter and a filled magenta circle. The filled green circle represents the blue object detected by DAOрнот inside the $3 \sigma$ match circle of 47 Tuc I. The filled orange circle is the same blue object detected by Ks 2 inside the $3 \sigma$ match circle of 47 Tuc I. Calibrated magnitudes were converted to absolute magnitudes using a distance to 47 Tuc of $4.69 \mathrm{kpc}$ (Woodley et al. 2012) and reddening $E(B-V)=0.04$ (Salaris et al. 2007). The lines correspond to cooling models for He WDs with thick $\mathrm{H}$ envelopes as in Serenelli et al. (2002), computed for an initial metallicity of $Z=0.003$. Labels at the top of each track represent the mass of the He WD in units of solar mass.

absolute magnitude in the F390W filter $\left(M_{B_{390}}\right)$ as the independent variable. Table 3 summarizes the results.

Cooling ages derived from our models (column 3 in Table 3) represent the time since the point of highest temperature in the cooling track until the observed location along the track. Prior to this time lapse is the time between Roche lobe detachment (when the mass transfer from the He WD progenitor to the neutron star stops) until the point where the highest $T_{\text {eff }}$ is reached. This phase lasts only a few Myr for masses $0.25 \mathrm{M}_{\odot}$ and higher, but can amount to $\sim 1.5 \mathrm{Gyr}$ for the $0.16 \mathrm{M}_{\odot}$ model, $\sim 1 \mathrm{Gyr}$ for $0.175 \mathrm{M}_{\odot}$, and $\sim 500 \mathrm{Myr}$ for $0.2 \mathrm{M}_{\odot}$. The exact value depends on the uncertain details of the binary evolution, and sets a lower limit to the total age. Istrate et al. (2014) have derived a general expression for the duration of this 'proto-WD' phase $\left(\Delta t_{\text {proto }}\right)$ in terms of the He WD mass, which for our MSP companions is given in the last column of Table 3. We find that the proto-WD time-scale in our models and the value of $\Delta t_{\text {proto }}$ are in general agreement.

We have estimated the number of He WD-like objects that we expect to find by chance within a separation of 0.016 arcsec (i.e. the largest offset among the suggested matches for 47 Tuc Q, S, T, $\mathrm{U}$, and Y; see Table 2) from the radio positions. To this end, we determined the density of blue objects (stars $\operatorname{arcsec}^{-2}$ ) enclosed by the cooling tracks for masses $0.16 \leq M_{\mathrm{WD}} / \mathrm{M}_{\odot} \leq 0.30$ and absolute magnitudes $6 \leq M_{B_{390}} \leq 11$ (see Fig. 2). For the total area of the $3 \sigma$ match circles of 19 MSPs, the expected number is very small, $9.5 \times 10^{-5}$. This suggests that our five identified MSP counterparts are indeed not chance coincidences.

For the binary MSP 47 Tuc I, we find a very blue object at $0.12 \operatorname{arcsec}(1.6 \sigma)$ with a $U_{300}-B_{390}$ colour that puts it on or very close to the CO WD sequence (Figs 2 and 4). This offset is much 


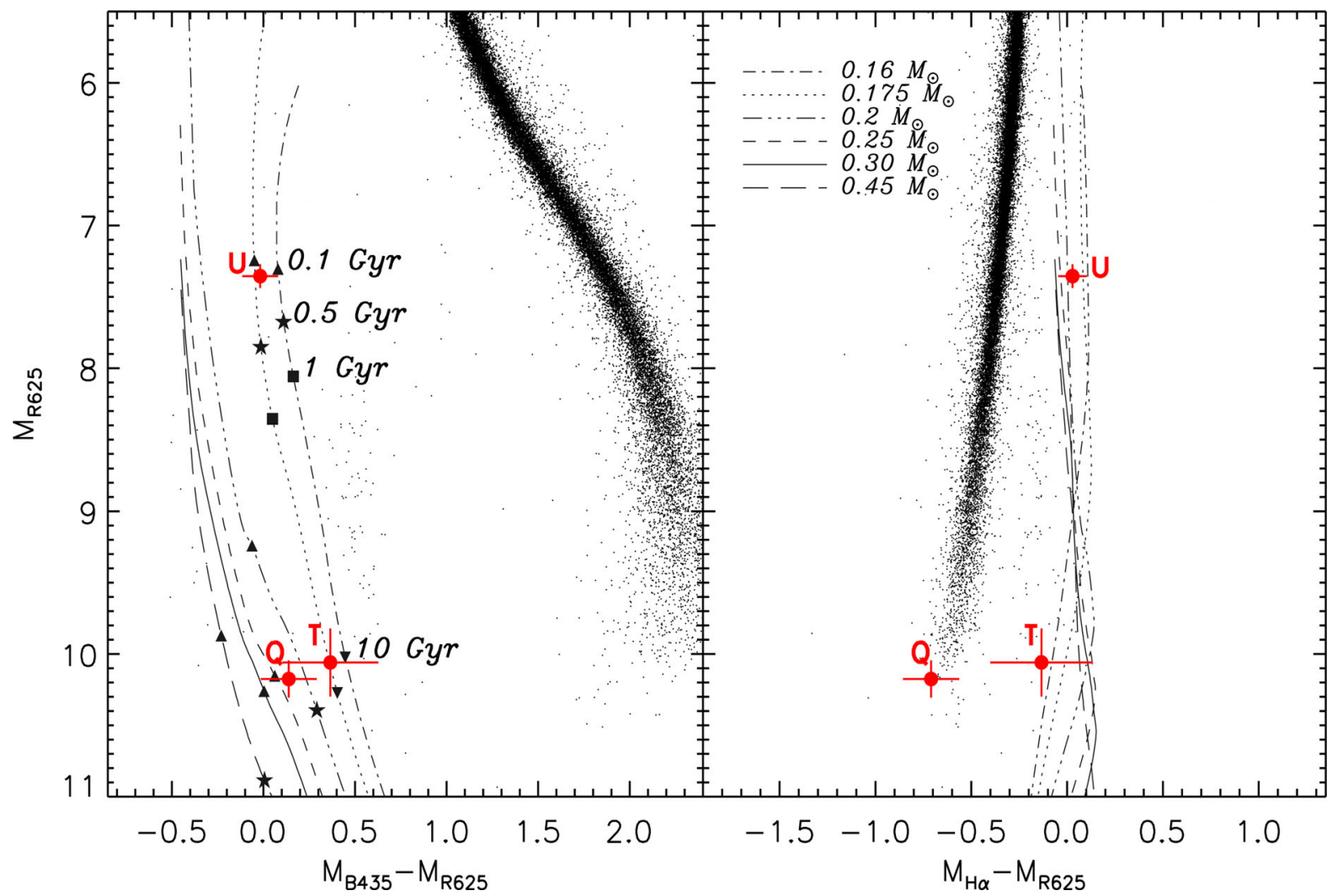

Figure 3. $B_{435}-R_{625}$ versus $R_{625}$ (left) and $\mathrm{H} \alpha-R_{625}$ versus $R_{625}$ (right) CMDs from our WFC DAOPHOT photometry. MSP counterparts are plotted with filled red circles. Calibrated Vega-mag magnitudes were converted to dereddened absolute magnitudes using a distance to 47 Tuc of $4.69 \mathrm{kpc}$ (Woodley et al. 2012) and reddening $E(B-V)=0.04$ (Salaris et al. 2007). We include He WD cooling tracks (Serenelli et al. 2002) for masses in the range 0.16-0.45 $\mathrm{M}_{\odot}$ and an initial metallicity of $Z=0.003$. Photometry errors include the DAOPHOT uncertainties, and errors in the distance modulus and $E(B-V)$.

Table 1. Parameters of the MSPs in 47 Tuc whose companions are discussed in this paper, for a reference epoch MJD 51600. Positions, spin periods $\left(P_{\mathrm{s}}\right)$, orbital periods $\left(P_{\mathrm{b}}\right)$, limits on companion masses $\left(m_{\mathrm{c}}\right.$, for an assumed neutron-star mass of $\left.1.35 \mathrm{M}_{\odot}\right)$, and characteristic ages $\left(\tau_{\mathrm{c}}\right)$ are from Freire et al. (in preparation). X-ray luminosities in the $0.1-$ $10 \mathrm{keV}$ band $\left(L_{\mathrm{X}}\right)$ were taken from Bogdanov et al. (2006). Units of right ascension are hours, minutes, and seconds, and units of declination are degrees, arcminutes, and arcseconds. Numbers in parentheses are $1 \sigma$ uncertainties in the last significant digit, errors in $P_{\mathrm{S}}$ are in 13th decimal, while errors in $P_{\mathrm{b}}$ are in the ninth decimal or smaller. Values of $\tau_{\mathrm{c}}$ are lower limits based on the $2 \sigma$ upper limit for the spin-period derivatives.

\begin{tabular}{llllllll}
\hline MSP & RA (J2000) & Dec. (J2000) & $\begin{array}{c}P_{\mathrm{s}} \\
(\mathrm{ms})\end{array}$ & $\begin{array}{c}P_{\mathrm{b}} \\
(\mathrm{d})\end{array}$ & $\begin{array}{c}m_{\mathrm{c}} \\
\left(\mathrm{M}_{\odot}\right)\end{array}$ & $\begin{array}{c}\tau_{\mathrm{c}} \\
(\mathrm{Gyr})\end{array}$ & $\begin{array}{c}L_{\mathrm{X}} \\
\left(10^{30} \mathrm{erg} \mathrm{s}^{-1}\right)\end{array}$ \\
\hline $\mathrm{Q}$ & $00: 24: 16.4903(2)$ & $-72: 04: 25.1653(8)$ & 4.03 & 1.189 & $>0.18$ & $>1.43$ & 3.9 \\
$\mathrm{~S}$ & $00: 24: 03.9794(1)$ & $-72: 04: 42.3535(5)$ & 2.83 & 1.201 & $>0.09$ & $>0.91$ & 8.9 \\
$\mathrm{~T}$ & $00: 24: 08.5487(7)$ & $-72: 04: 38.928(3)$ & 7.58 & 1.126 & $>0.17$ & $>0.32$ & 2.9 \\
$\mathrm{U}$ & $00: 24: 09.83626(7)$ & $-72: 03: 59.6889(3)$ & 4.34 & 0.429 & $>0.12$ & $>1.91$ & 5.1 \\
$\mathrm{Y}$ & $00: 24: 01.4016(3)$ & $-72: 04: 41.837(1)$ & 2.19 & 0.521 & $>0.14$ & $>2.2$ & 3.9 \\
\hline
\end{tabular}

larger, and its colour much bluer, than those for the five binary MSPs discussed above. We estimate the probability that this source is a spurious match by considering that blue $\left(U_{300}-B_{390} \lesssim-0.65\right)$ objects are found in the error circles of two (47 Tuc D and N) of the seven isolated MSPs in the UVIS field. Due to their intrinsic faintness in the NUV/optical, no counterparts are expected for the isolated MSPs. The blue objects close to 47 Tuc D [at 0.14 arcsec or $1.8 \sigma$, and at $(-1.44,11.50)$ in the CMD of Fig. 2] and 47 Tuc $\mathrm{N}$ [at 0.16 arcsec or $2.1 \sigma$, and at $(-0.65,9.68)$ in the CMD] are therefore almost certainly chance alignments. The probability to find a blue star in the $3 \sigma$ error circle by chance in a single trial is therefore, approximately, $2 / 7 \approx 0.29$. This results in a (binomial) probability to find a blue random match for exactly one of ten ${ }^{10}$ binary MSPs in our field of $\sim 14$ per cent. While we consider the NUV identification of 47 Tuc I not very likely, we give more details on this object in Section 4.6, and provide a finding chart in Fig. 4.

For 47 Tuc E, H, J (binaries), and L (isolated), not a single NUV object was detected inside their $3 \sigma$ match circles. For 47 Tuc O, R, and $\mathrm{W}$ (binaries) and C, F, G, and M (isolated), the objects in the

\footnotetext{
${ }^{10}$ For 47 Tuc $\mathrm{U}$ and $\mathrm{W}$, excellent counterparts have already been identified.
} 
Table 2. Astrometric and photometric results for the MSP companions discussed in this paper. Errors in the absolute astrometry of the celestial positions are $0.074 \operatorname{arcsec}(1 \sigma)$. Units of right ascension are hours, minutes, and seconds, and units of declination are degrees, arcminutes, and arcseconds. Columns 4 to 8 give the calibrated photometry on the Vega-mag system and the errors from DAорнот. The projected distance of the companions from the cluster centre $\left(\delta_{\mathrm{c}}\right)$ was calculated using the coordinates of the centre of 47 Tuc as reported by Goldsbury et al. (2011). The offsets between the X-ray and NUV positions (last column) were determined using the positions from H05. The value of $\sigma_{\mathrm{X}}$ is the combined error in the NUV and X-ray positions.

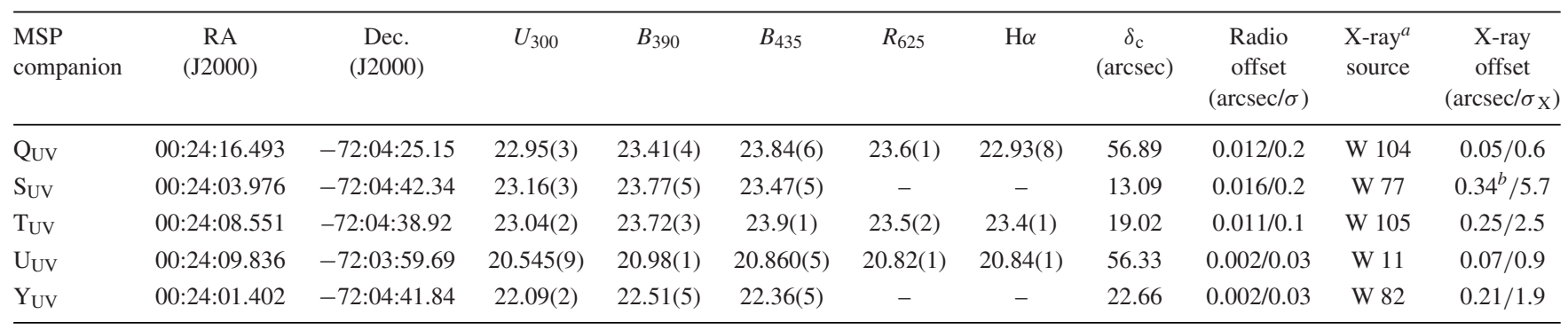

${ }^{a}$ Using the nomenclature in $\mathrm{H} 05$.

${ }^{b}$ The X-ray position used for 47 Tuc $\mathrm{S}$ corresponds to the position of a single detected X-ray source that is the combination of X-ray emission from 47 Tuc $\mathrm{F}$ and 47 Tuc S. This explains the apparent large X-ray astrometric offset.

Table 3. Limits on companion masses ( $m_{\mathrm{c}}$, for an assumed neutron-star mass of $1.35 \mathrm{M}_{\odot}$ ) as derived from the mass function (Freire et al., in preparation). Masses $\left(M_{\mathrm{WD}}\right)$, cooling ages, effective temperatures $\left(T_{\text {eff }}\right)$, surface gravities $(\log g)$, and luminosities $(L)$ for the MSP companions studied, as derived from our NUV photometry and our $Z=0.003 \mathrm{He}$ WD cooling models. In column 8 , the inclination angle (i) as inferred from the mass function and the estimated masses $M_{\mathrm{WD}}$. The last column gives $\Delta t_{\text {proto }}$, which is the lapse of time from the Roche lobe detachment until the proto-He WD reaches the WD cooling track, calculated using the relation derived by Istrate et al. (2014).

\begin{tabular}{lcccccccc}
\hline Companion & $m_{\mathrm{c}}\left(\mathrm{M}_{\odot}\right)$ & $M_{\mathrm{WD}}\left(\mathrm{M}_{\odot}\right)$ & $\operatorname{Age}^{a}(\mathrm{Gyr})$ & $T_{\text {eff }}(\mathrm{kK})$ & $\log g$ & $\log L\left(\mathrm{~L}_{\odot}\right)$ & $i\left(^{\circ}\right)$ & $\Delta t_{\text {proto }}(\mathrm{Gyr})$ \\
\hline $\mathrm{Q}_{\mathrm{UV}}$ & $>0.18$ & $0.175-0.20$ & $5.2-0.3$ & $8.9-10.6$ & $6.4-6.7$ & -1.9 to -1.8 & $90-63$ & $1-0.4$ \\
$\mathrm{~S}_{\mathrm{UV}}$ & $>0.09$ & $0.20-0.25$ & $0.4-0.1$ & $9.7-11.3$ & $6.7-7$ & -2.0 to -1.9 & $28-22$ & $0.4-0.1$ \\
$\mathrm{~T}_{\mathrm{UV}}$ & $>0.17$ & $0.20-0.30$ & $0.4-0.1$ & $9.9-12.8$ & $6.7-7.2$ & -2.0 to -1.8 & $58-36$ & $0.4-0.02$ \\
$\mathrm{U}_{\mathrm{UV}}$ & $>0.12$ & $0.16-0.175^{b}$ & $0.2-0.3$ & $10.1-12$ & $5.5-5.8$ & -0.9 to -0.8 & $51-46$ & $1.9-1.3$ \\
$\mathrm{Y}_{\mathrm{UV}}$ & $>0.14$ & $0.16-0.175$ & $2.1-1.8$ & $8.9-10$ & $6-6.2$ & -1.6 to -1.5 & $60-53$ & $1.9-1.3$ \\
\hline
\end{tabular}

${ }^{a}$ Ages represent the time between the point of highest $T_{\text {eff }}$ on the cooling track until the observed point along the track.

${ }^{b}$ An upper mass limit of $0.17 \mathrm{M}_{\odot}$ was found by E01, for which they estimated an age of $\sim 0.6 \mathrm{Gyr}, T_{\text {eff }}=11000 \mathrm{~K}$, and log $g=5.6$.
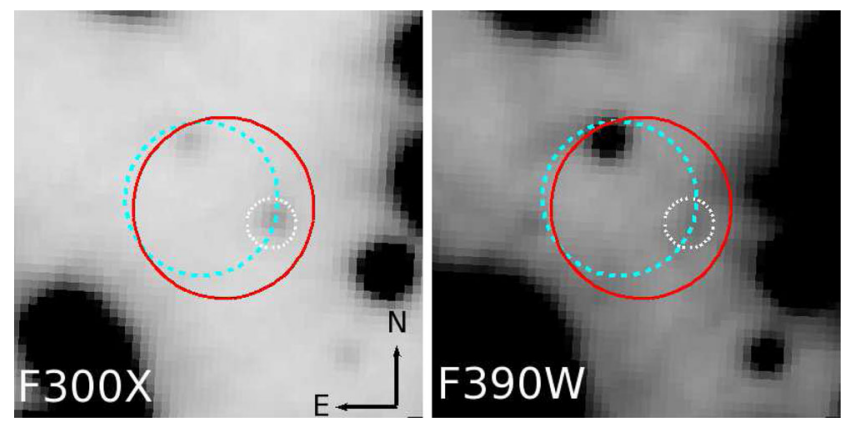

Figure 4. Finding chart of 47 Tuc I in the NUV. The solid red circles are the $3 \sigma$ match circles for the radio position of the pulsar. They are centred on 47 Tuc I. The dashed cyan circles are the $3 \sigma$ match circles of the Chandra source. The dashed white circles indicate the blue object that lies at $0.12 \operatorname{arcsec}$ from the pulsar. The images are $1.0 \operatorname{arcsec} \times 1.0 \operatorname{arcsec}$ in size.

match circles have colours that place them on the main sequence or sub-giant branch of 47 Tuc and lie at least $0.11 \operatorname{arcsec}(1.4 \sigma)$ away from the radio positions. Without any additional information, we cannot conclusively say if any of these are likely counterparts, and we do not discuss them further in this paper. We note that the true, non-degenerate, counterpart to 47 Tuc W (Edmonds et al. 2002) is not detected in the NUV images. This is not unexpected as it is relatively faint (mean $V=22.3$ ) and red (compared to $\mathrm{Q}_{\mathrm{UV}}, \mathrm{S}_{\mathrm{UV}}$, $\mathrm{T}_{\mathrm{UV}}, \mathrm{U}_{\mathrm{UV}}$, and $\mathrm{Y}_{\mathrm{UV}}$ ).

\subsection{Tuc $Q$}

In the CMD of Fig. 2, the counterpart of 47 Tuc Q is located between the main sequence of the Small Magellanic Cloud (SMC) and the CO WD cooling sequence, at a similar $U_{300}-B_{390}$ colour as $\mathrm{U}_{\mathrm{UV}}$, but about 2.4 mag fainter in $B_{390}$. E03a reported the presence of a variable main-sequence turnoff star very close (at 0.24 arcsec) to the position of 47 Tuc Q. This star, which they labelled 'nQ', is the bright object to the south-west of Quv in Fig. 1. They discarded nQ as the true counterpart to the MSP, since its period of optical variability does not match the orbital period of the pulsar derived from radio timing. The faint blue star $\mathrm{Q}_{\mathrm{UV}}$ lies closer to the radio position than nQ $(0.012$ arcsec or $0.2 \sigma)$, but was not detected by E03a. It lies in the PSF wings of two stars (nQ and a star on the 47 Tuc sub-giant branch), which makes $\mathrm{Q}_{\mathrm{UV}}$ difficult to detect at optical wavelengths.

In our $R_{625}$ versus $B_{435}-R_{625} \mathrm{CMD}, \mathrm{Q}_{\mathrm{Uv}}$ also lies in the region where He WDs are expected. But unlike the counterparts to 47 Tuc $\mathrm{U}$ and $\mathrm{T}$ that lie to the red side ( $\mathrm{H} \alpha$ faint side) of the main sequence in the $R_{625}$ versus $\mathrm{H} \alpha-R_{625} \mathrm{CMD}$ (see Sections 4.3 and 4.5, and Fig. 3), there is no indication for the presence of a broad $\mathrm{H} \alpha$ absorption line in $\mathrm{Q}_{\mathrm{Uv}}$ : its $\mathrm{H} \alpha-R_{625}$ colour is consistent with the colour of most other stars in the field with similar $R_{625}$ magnitude. If $\mathrm{Q}_{\mathrm{UV}}$ actually is an $\mathrm{He} \mathrm{WD}$, the $\mathrm{H} \alpha-R_{625}$ colour could be explained if there is some residual $\mathrm{H} \alpha$ emission in the system that effectively fills in the $\mathrm{H} \alpha$ absorption line of the $\mathrm{H}$-rich outer layer of the WD. From the location of $\mathrm{Q}_{\mathrm{Uv}}$ in a $B_{625}-R_{625}$ versus $\mathrm{H} \alpha-R_{625}$ colour-colour diagram and synthetic colours of 
simulated spectra, we estimate that the equivalent width of such an emission line is about $-70 \AA$. However, the inclination inferred from the mass function (Table 1) and our estimate for $M_{\mathrm{WD}}$ (Table 3 ) is high $\left(63^{\circ}-90^{\circ}\right)$, so eclipses are expected if a large cloud of ionized material would be present. The absence of radio eclipses in 47 Tuc Q then argues against the presence of such an extended $\mathrm{H} \alpha$ emission region. We note that, given the proximity of the two brighter stars, the photometry for QUV should be considered with some caution; in particular, the PSFs of relatively bright stars in the $\mathrm{F} 625 \mathrm{~W}$ and $\mathrm{H} \alpha$ images show airy disc peaks around the central region of the PSF, which coincide with the location of QUV and could affect its colours if not properly modelled.

The location of Quv with respect to the He WD cooling tracks suggests a mass between $\sim 0.175$ and $0.20 \mathrm{M}_{\odot}$. This is consistent with the lower limit of the companion mass derived from the pulsar mass function $\left(0.18 \mathrm{M}_{\odot}\right.$ for an assumed neutron-star mass of $\left.1.35 \mathrm{M}_{\odot}\right)$. The derived cooling age is very uncertain, as $\mathrm{Q}_{\mathrm{UV}}$ lies in between the tracks for the slowly cooling $0.175 \mathrm{M}_{\odot}$ models and the rapidly cooling $0.20 \mathrm{M}_{\odot}$ He WDs; these respective masses predict an age range of 5.2-0.3 Gyr. In addition, the proto-WD phase can add up to $1 \mathrm{Gyr}$ to obtain the time that passed since Roche lobe detachment. However, a comparison of the WD age with the characteristic pulsar spin-down age $\tau_{\mathrm{c}}$ is difficult. The observed period derivatives for the 47 Tuc MSPs are dominated by the effect of the acceleration of the pulsars along the line of sight in the gravitational potential of the cluster. As a result, the intrinsic period derivatives, and therefore the pulsar characteristic ages, are poorly constrained. However, better estimations of the intrinsic period derivatives, for stable MSP- WD systems, can be made by using the time derivative of the observed orbital periods, which essentially measures the acceleration of each binary MSP along the line of sight. Details about this technique will be given in Freire et al. (in preparation). By using this method, we obtain $\tau_{\mathrm{c}}>1.43 \mathrm{Gyr}$ for $\mathrm{Quv}_{\mathrm{uv}}$.

\subsection{Tuc $S$}

$\mathrm{S}_{\mathrm{UV}}$, together with $\mathrm{T}_{\mathrm{UV}}$, is the faintest MSP companion that we found. This object is clearly visible in our F435W images, but in the $\mathrm{F} 625 \mathrm{~W}$ and $\mathrm{H} \alpha$ images, a reliable detection cannot be made. This is partly the result of a relatively bright diffraction spike of a brighter star that passes within a few pixels of the centre of the PSF of $\mathrm{S}_{\mathrm{UV}}$. From comparison with the He WD cooling tracks, we derive a mass of about $0.2-0.25 \mathrm{M}_{\odot}$ and an age of about 0.4 0.1 Gyr. Taking into account its time spent as a 'proto-WD' (up to $0.4 \mathrm{Gyr}$ ), this age range is roughly consistent with the lower limit on the pulsar characteristic age.

47 Tuc $\mathrm{S}$ and the isolated pulsar 47 Tuc F are only 0.74 arcsec apart. Both are associated with a single Chandra source, W 77, which is likely a blend of (at least) these two sources. This explains the relatively large offset between $\mathrm{S}_{\mathrm{UV}}$ and $\mathrm{W} 77$ in Table 2.

\subsection{Tuc $T$}

We derive a mass for $\mathrm{T}_{\mathrm{UV}}$ of $0.2-0.3 \mathrm{M}_{\odot}$, in agreement with the lower limit of the companion mass (Table 1). The approximate total WD age is about $0.8-0.1 \mathrm{Gyr}$. This value is roughly consistent with the lower limit on $\tau_{\mathrm{c}}$ of this MSP.

$\mathrm{T}_{\mathrm{UV}}$ lies in between the CO WD cooling sequence and the SMC main sequence in our NUV CMD. The blue colour of $\mathrm{T}_{U V}$ is apparent from the finding charts in Fig. 1. In the F390W image, $\mathrm{T}_{U V}$ has a close neighbour at a separation of only 0.07 arcsec. In the F300X image, the neighbour, as well as stars of similar F390W magnitude, is much fainter. $\mathrm{T}_{\mathrm{UV}}$ is clearly detected in our $\mathrm{F} 435 \mathrm{~W}$ images. While also detected in $\mathrm{F} 625 \mathrm{~W}$ and $F 658 N$, it is very faint at these redder wavelengths; the close neighbour and the contamination of its PSF by a weak diffraction spike of another neighbouring star lead to relatively large errors in its optical colours (Fig. 3). Nevertheless, the optical counterpart is also clearly blue, and its location on the red side of the $\mathrm{H} \alpha-R_{625}$ main sequence points at the presence of a strong $\mathrm{H} \alpha$ absorption line. $\mathrm{T}_{\mathrm{UV}}$ was already identified as the likely counterpart to 47 Tuc $\mathrm{T}$ by E03a. They detected $\mathrm{T}_{\mathrm{UV}}$ in their $U$ images but not in $V$ and $I$, and could therefore only derive an upper limit to the colour.

\subsection{Tuc $Y$}

Our photometry confirms the blue colour of $\mathrm{Y}_{\mathrm{UV}}$ reported by E03a. We estimate that the mass of $\mathrm{Y}_{\mathrm{UV}}$ is between 0.16 and $0.175 \mathrm{M}_{\odot}$, in agreement with the lower limit from the radio mass function (Table 1). The total age estimate, about $2 \mathrm{Gyr}$ for the cooling time plus 1.3-1.9 Gyr for the proto-WD phase, makes $\mathrm{Y}_{\mathrm{UV}}$, possibly together with $\mathrm{Q}_{\mathrm{UV}}$, the oldest of the five WD companions discussed in this paper.

$\mathrm{Y}_{\mathrm{UV}}$ is clearly detected in our F435W image. The PSF of the neighbouring bright object, which can be seen a little offset from the centre in the finding charts of Fig. 1, reduces the sensitivity for very faint objects. As a result, $\mathrm{Y}_{\mathrm{UV}}$ is not detected in the $R_{625}$ and $\mathrm{H} \alpha$ images.

\subsection{Tuc $U$}

E01 found that $\mathrm{U}_{\mathrm{UV}}$ is a variable blue object, with a semi-amplitude variation of $0.004 \mathrm{mag}$ and with a period that is consistent with the orbital period derived from radio timing (about $0.43 \mathrm{~d}$ ). Based on a comparison between the $U, V$, and $I$ photometry for this star and the Serenelli et al. (2001) models for He WD evolution, its mass and age were estimated to be $\sim 0.17 \mathrm{M}_{\odot}$ and $\sim 0.6 \mathrm{Gyr}$, respectively. E01 also identified this object as the counterpart of the X-ray source W11. E01 indeed found that the He WD companion is well inside its Roche lobe, which also indicates that there is no ongoing accretion that contributes to the X-rays.

$\mathrm{U}_{\mathrm{UV}}$ is the brightest of the five counterparts discussed in this work. It is an isolated object, so its UVIS and WFC photometry is robust. $\mathrm{U}_{\mathrm{UV}}$ is located above and to the left of the SMC main sequence in the NUV and optical CMDs, in agreement with the results of E01 and E03a. The location of $\mathrm{U}_{\mathrm{UV}}$ to the red side of the main sequence in the $R_{625}$ versus $\mathrm{H} \alpha-R_{625}$ CMD points at the presence of a strong $\mathrm{H} \alpha$ absorption line, and is indeed in agreement with the He WD cooling tracks.

The He WD atmosphere models used in this paper are computed for a lower metallicity and make use of updated atmosphere models (Rohrmann, Althaus \& Kepler 2011) with respect to the ones used in E01. From the updated models and our UVIS photometry, we constrain the mass for $\mathrm{U}_{\mathrm{UV}}$ to be $0.16-0.175 \mathrm{M}_{\odot}, T_{\mathrm{eff}} \approx 11000 \mathrm{~K}$, and $\log g \approx 5.6$. This is consistent with the results from our WFC photometry and with the parameters derived by E01. Our cooling age estimate $(0.2-0.3 \mathrm{Gyr})$ is somewhat lower than that found in the latter study ( $\sim 0.6 \mathrm{Gyr})$. If we take into account $\Delta t_{\text {proto }}$, the total age becomes 2.1-1.6 Gyr; this is still consistent with the lower limit to $\tau_{\mathrm{c}}$ from Table 1 . 


\subsection{Tuc I}

The blue star in the $3 \sigma$ error circle of 47 Tuc I is very faint. The errors in the photometry are considerable, and we plot the results from both DAOPHOT and $\mathrm{KS} 2$ in Fig. 2 (denoted as oI and $\mathrm{oI}_{\mathrm{KS} 2}$, respectively); it is too faint to be detected in any of our optical images. This object is too blue to be an He WD. While the colours are more consistent with a CO WD than an He WD, the former option is not likely if the star is truly associated with 47 Tuc I. MSPs with CO WD companions typically have longer spin periods compared to systems with He WD companions (Tauris, Langer \& Kramer 2012; note the exception of PSR J1614-2230). The spin period of 47 Tuc I, on the other hand, is very short $\left(P_{\mathrm{s}}=3.2 \mathrm{~ms}\right.$; Freire et al. 2003). We also point out that the mass function suggests that the companion is a very low mass object, with $m_{\mathrm{c}}>0.013 \mathrm{M}_{\odot}$. If the blue object is the $\sim 0.5 \mathrm{M}_{\odot} \mathrm{CO}$ WD companion to 47 Tuc I, this would imply an inclination of $i \approx 1.8$. None of the aforementioned arguments are conclusive grounds for rejecting or confirming the association.

\section{DISCUSSION}

The five binary MSPs in 47 Tuc that we discuss in this work are characterized by spin periods below $8 \mathrm{~ms}$, orbital periods $P_{\mathrm{b}}$ between 0.43 and $1.2 \mathrm{~d}$, and companions more massive than $\sim 0.09 \mathrm{M}_{\odot}$ (Table 1). Based on a compilation of available data for binary pulsars in the Galactic disc, the nature of the companion for such binary pulsars is most likely an He WD (see Tauris et al. 2012). We find that our NUV/optical photometry for the five MSP counterparts is indeed consistent with the magnitudes and colours predicted by the low-metallicity He WD cooling models by Serenelli et al. (2002). In particular, the derived mass constraints lie in the range 0.16$0.30 \mathrm{M}_{\odot}$. Our results for the companion of 47 Tuc $\mathrm{U}$ are consistent with those derived by E01, who compared $U, V$, and $I$ photometry for this star with the solar-metallicity He WD models by Serenelli et al. (2001).

The standard formation scenario for an MSP accompanied by an He WD involves a long period of stable mass transfer. In the models by Serenelli et al. (2002), the He WDs emerge from this phase with a thick $\mathrm{H}$ envelope. Calculations for the subsequent evolution of WDs predict a dichotomy in cooling behaviour ${ }^{11}$ (e.g. Alberts et al. 1996; Althaus, Serenelli \& Benvenuto 2001; Serenelli et al. 2001; Panei et al. 2007; Althaus et al. 2013). He WDs with masses $\gtrsim 0.2 \mathrm{M}_{\odot}$ (or equivalently $P_{\mathrm{b}} \gtrsim 1.55$ d; see van Kerkwijk et al. 2005) experience thermonuclear $\mathrm{H}$-shell flashes before they reach the cooling track. These flashes leave the WD with a thin $\mathrm{H}$ envelope, as a result of additional diffusion-induced $\mathrm{H}$-shell flashes. After these short-lived episodes, the WD remnant cools down on a relatively short timescale. In He WDs less massive than $\sim 0.2 \mathrm{M}_{\odot}, \mathrm{H}$-shell flashes do not occur, and therefore a thick $\mathrm{H}$ envelope is retained. Residual $\mathrm{H}$ burning can keep up a high temperature over long time-scales, resulting in much longer cooling times. Fig. 2 implies that $S_{U V}$ and $\mathrm{T}_{\mathrm{UV}}$ cool fast, $\mathrm{U}_{\mathrm{UV}}$ and $\mathrm{Y}_{\mathrm{UV}}$ cool slowly, while for $\mathrm{Q}_{\mathrm{UV}}$ the time-scale is ambiguous. Unfortunately, the spin-down ages of the pulsars in 47 Tuc are too uncertain to give an independent constraint on the WD age, and hence, mass.

\footnotetext{
${ }^{11}$ The evolutionary models by Istrate et al. (2014) do not show this dichotomy in cooling behaviour depending on the occurrence of H-shell flashes or not, but rather indicate that the cooling time-scale mainly depends on the mass of the proto-WD.
}

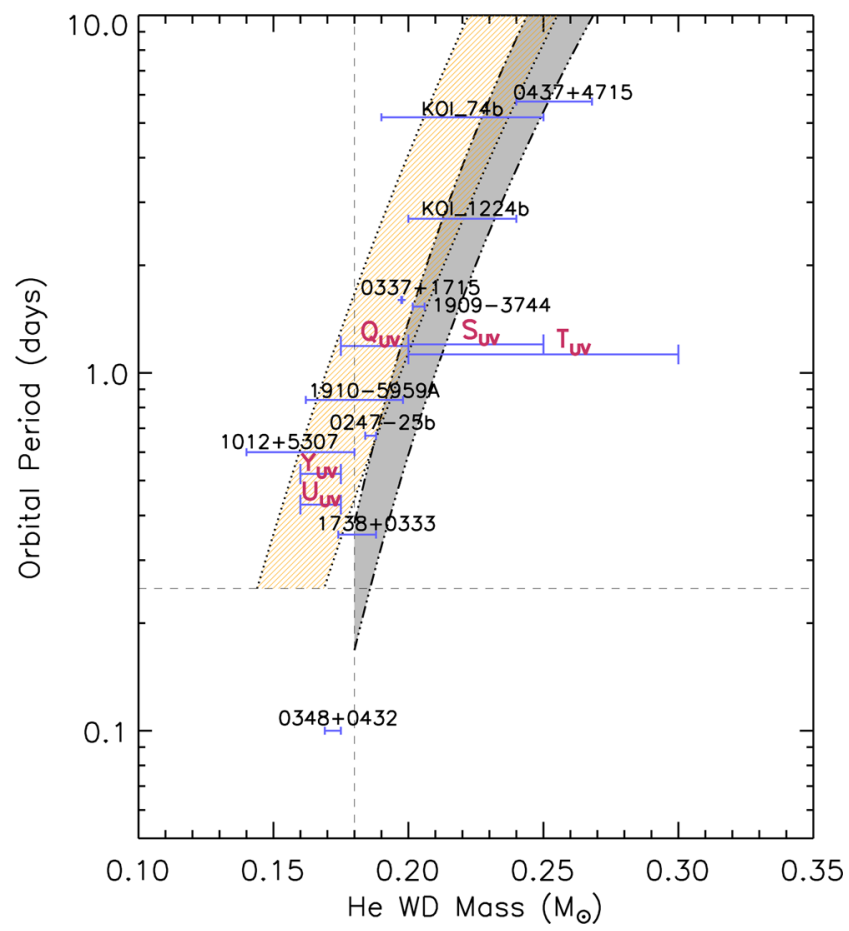

Figure 5. Mass-orbital period relations for He WDs. In grey is the relation as calculated by Tauris \& Savonije (1999), with metallicities in the range $Z=0.001-0.02$ (from right to left) and for WD masses in the range 0.18$0.45 \mathrm{M}_{\odot}$. In orange is the relation by De Vito \& Benvenuto (2010) for solar metallicity and for orbital periods $>0.25 \mathrm{~d}$. The width of the relation corresponds to the uncertainties in its parameters. Overplotted are our results for the companions of 47 Tuc Q, S, T, U, and Y. The error bars denote the possible masses for each object as determined from the WD cooling tracks by Serenelli et al. (2002). We also plot other pulsar companions for which $1 \sigma$ uncertainties are less than 10 per cent of the WD mass (see Tauris \& van den Heuvel 2014 and references therein). The dashed vertical line denotes the lower mass limit $\left(0.18 \mathrm{M}_{\odot}\right)$ for which the Tauris \& Savonije (1999) relation is valid. The dashed horizontal line shows the minimum orbital period $(0.25 \mathrm{~d})$ for which the De Vito \& Benvenuto (2010) relation is valid.

There are only two binary MSPs in our field whose mass functions suggest He WD-like companions but for which we have not found a plausible counterpart, namely 47 Tuc $\mathrm{E}$ and $\mathrm{H}$. Their orbital periods are the longest of the binary MSPs in 47 Tuc included in our field ( $\sim 2.3 \mathrm{~d}$ ). The relation between orbital period and companion mass (see for example Tauris \& Savonije 1999) then suggests that the companions in these systems are more massive than those in the other MSPs. Since a higher mass implies a shorter cooling time, the non-detection of the companions to 47 Tuc $\mathrm{E}$ and $\mathrm{H}$ can be understood if they were formed more than $\sim 0.7 \mathrm{Gyr}$ ago so that they have dimmed below the detection limit.

In Fig. 5, we show the relation between the mass of the $\mathrm{He}$ WD and the orbital period based on binary-evolution calculations by Tauris \& Savonije (1999), valid for He WDs masses between 0.18 and $0.45 \mathrm{M}_{\odot}$ and De Vito \& Benvenuto (2010), valid for $P_{\mathrm{b}}>0.25 \mathrm{~d}$. We find that the orbital periods and mass estimates for $\mathrm{S}_{\mathrm{UV}}$ and $\mathrm{T}_{\mathrm{UV}}$ are more consistent with the relation derived by Tauris \& Savonije (1999). In contrast, $Y_{U V}$ and $U_{U V}$ are offset from that relation, but their estimated masses lie below the mass range to which the relation applies. However, we observe that $\mathrm{Y}_{\mathrm{UV}}$ and $\mathrm{U}_{\mathrm{UV}}$ are in good agreement with the relation by De Vito \& Benvenuto (2010). QUv is consistent with both relations. De Vito \& Benvenuto (2010) made different assumptions on the mass-transfer process 
and they obtain, for the same orbital periods, lower masses for the WD companions than Tauris \& Savonije (1999). They also find that their mass-orbital period relation is not sensitive to the initial mass of the accreting neutron star. Studies about the assumption on how conservative the mass transfer is have found that the dispersion around the De Vito \& Benvenuto (2010) relation decreases as the He WD mass increases (De Vito \& Benvenuto 2012).

The five binary MSPs discussed here have X-ray luminosities $L_{\mathrm{X}}=2.9-8.9 \times 10^{30} \mathrm{erg} \mathrm{s}^{-1}$ (Bogdanov et al. 2006). Like for the majority of the 47 Tuc MSPs, it was found that their X-ray emission can be described by a thermal (blackbody or neutron-star hydrogen atmosphere) spectrum as expected for emission from the heated magnetic polar caps of the neutron stars. Using the $L, T_{\text {eff }}$, and mass estimates from Table 3, and the orbital periods from Table 1, we have calculated the WD radii $\left(R_{\mathrm{WD}}\right)$ and (assuming a neutron-star mass of $\left.1.35 \mathrm{M}_{\odot}\right)$ the corresponding radii of the Roche lobe $\left(R_{\mathrm{L}, \mathrm{WD}}\right.$, using the formula of Paczyński 1971). We indeed find that all five WD companions fit well within their Roche lobe $\left(R_{\mathrm{L}, \mathrm{WD}} / R_{\mathrm{WD}} \gtrsim 5\right)$ as already found for 47 Tuc $U$ by E01, so we indeed expect that accretion does not contribute to the X-ray luminosity.

For the five systems we studied, we calculate the contribution of the MSP radiation to the observed NUV luminosity of the WD. We used the values of the spin-down luminosity $(\dot{E})$ for each MSP given by Bogdanov et al. (2006). Assuming isotropic emission from the neutron star, we calculate the energy flux intercepted by the $\operatorname{WD}\left(\dot{E}_{i}\right)$ using our estimates of $R_{\mathrm{WD}}$ and the separation between the stars. For $\mathrm{Q}_{\mathrm{UV}}, \mathrm{S}_{\mathrm{UV}}$, and $\mathrm{T}_{\mathrm{UV}}$, we obtain $\log \dot{E}_{i}\left(\mathrm{~L}_{\odot}\right)<-4$ which is well below their NUV luminosities. This result indicates that irradiation is not driving the luminosities of these systems. On the other hand, for $\mathrm{Y}_{\mathrm{UV}}$ and $\mathrm{U}_{\mathrm{UV}}$, we obtain $-4.2<\log \dot{E}_{i}\left(\mathrm{~L}_{\odot}\right)<-2.3$ which is a closer value to their respective $L$, implying that heating might be present, though not dominating the NUV luminosity. This result can be understood if we take into account that these two companions have larger $R_{\mathrm{WD}}$ and are closer to their MSPs than the other three WDs.

We note that all the companions have cooling ages $\lesssim 6$ Gyr (including age uncertainties), which are considerably less than the age of the cluster $(9.9 \pm 0.7 \mathrm{Gyr}$; Hansen et al. 2013). Rasio, Pfahl \& Rappaport (2000) proposed that the formation of recycled MSP systems happened mostly during the early life of the cluster. However, our results may suggest that dynamical interactions during the later evolution of the cluster have a more important role in the formation of these systems (for an evolutionary scenario in this context, see for example Fregeau 2008).

The orbital periods of the five binary MSPs studied in this paper are all longer than the time span of the NUV observations $(\sim 9 \mathrm{~h})$; light curves constructed from these data alone give incomplete phase coverage. We defer a variability study, based on these and other HST data sets, to a future paper.

\section{ACKNOWLEDGEMENTS}

We appreciate the comments by the anonymous referee, which improved this work. LER-S acknowledges support from a CONACyT fellowship. We thank Dr. B. Lanzoni for pointing out minor mistakes in Table 2 in an earlier version of the paper.

\section{REFERENCES}

Alberts F., Savonije G. J., van den Heuvel E. P. J., Pols O. R., 1996, Nature, 380,676

Alpar M. A., Cheng A. F., Ruderman M., Shaham J., 1982, Nature, 300, 728 Althaus L. G., Serenelli A. M., Benvenuto O. G., 2001, MNRAS, 323, 471
Althaus L. G., Miller Bertolami M. M., Córsico A. H., 2013, A\&A, 557, A19

Anderson J. et al., 2008, AJ, 135, 2055

Bassa C. G., Verbunt F., van Kerkwijk M. H., Homer L., 2003, A\&A, 409, L31

Bogdanov S., Grindlay J. E., van den Berg M., 2005, ApJ, 630, 1029

Bogdanov S., Grindlay J. E., Heinke C. O., Camilo F., Freire P. C. C., Becker W., 2006, ApJ, 646, 1104

Cadelano M. et al., 2015, ApJ, 807, 91

Camilo F., Lorimer D. R., Freire P., Lyne A. G., Manchester R. N., 2000, ApJ, 535, 975

Clark G. W., 1975, ApJ, 199, L143

Cocozza G., Ferraro F. R., Possenti A., Beccari G., Lanzoni B., Ransom S., Rood R. T., D'Amico N., 2008, ApJ, 679, L105

De Vito M. A., Benvenuto O. G., 2010, MNRAS, 401, 2552

De Vito M. A., Benvenuto O. G., 2012, MNRAS, 421, 2206

Edmonds P. D., Gilliland R. L., Heinke C. O., Grindlay J. E., Camilo F., 2001, ApJ, 557, L57 ( E01)

Edmonds P. D., Gilliland R. L., Camilo F., Heinke C. O., Grindlay J. E., 2002, ApJ, 579, 741

Edmonds P. D., Gilliland R. L., Heinke C. O., Grindlay J. E., 2003a, ApJ, 596, 1177 ( E03a)

Edmonds P. D., Gilliland R. L., Heinke C. O., Grindlay J. E., 2003b, ApJ, 596, 1197

Ferraro F. R., Possenti A., D’Amico N., Sabbi E., 2001, ApJ, 561, L93

Ferraro F. R., Possenti A., Sabbi E., D’Amico N., 2003, ApJ, 596, L211

Fregeau J. M., 2008, ApJ, 673, L25

Freire P. C. C., 2013, in van Leeuwen J., ed., Proc. IAU Symp. 291, Neutron Stars and Pulsars: Challenges and Opportunities after 80 Years. Cambridge Univ. Press, Cambridge, p. 243

Freire P. C., Camilo F., Kramer M., Lorimer D. R., Lyne A. G., Manchester R. N., D'Amico N., 2003, MNRAS, 340, 1359

Goldsbury R., Richer H. B., Anderson J., Dotter A., Sarajedini A., Woodley K., 2011, AJ, 142, 66

Gonzaga S., Hack W., Fruchter A., Mack J., 2012, The DrizzlePac Handbook. STScI, Baltimore

Grindlay J. E., Heinke C., Edmonds P., Murray S. S., 2001, Science, 292, 2290

Hansen B. M. S. et al., 2013, Nature, 500, 51

Harris W. E., 1996, AJ, 112, 1487

Heinke C. O., Grindlay J. E., Edmonds P. D., Cohn H. N., Lugger P. M., Camilo F., Bogdanov S., Freire P. C., 2005, ApJ, 625, 796 (H05)

Istrate A. G., Tauris T. M., Langer N., Antoniadis J., 2014, A\&A, 571, L3

Manchester R. N., Lyne A. G., Johnston S., D’Amico N., Lim J., Kniffen D. A., 1990, Nature, 345, 598

Manchester R. N., Lyne A. G., Robinson C., Bailes M., D’Amico N., 1991, Nature, 352, 219

Paczyński B., 1971, ARA\&A, 9, 183

Pallanca C. et al., 2010, ApJ, 725, 1165

Pallanca C., Dalessandro E., Ferraro F. R., Lanzoni B., Beccari G., 2013, ApJ, 773, 122

Pallanca C., Ransom S. M., Ferraro F. R., Dalessandro E., Lanzoni B., Hessels J. W. T., Stairs I., Freire P. C. C., 2014, ApJ, 795, 29

Panei J. A., Althaus L. G., Chen X., Han Z., 2007, MNRAS, 382, 779

Ransom S. M., 2008, in Vesperini E., Giersz M., Sills A., eds, Proc. IAU Symp. 246, Dynamical Evolution of Dense Stellar Systems. Cambridge Univ. Press, Cambridge, p. 291

Rasio F. A., Pfahl E. D., Rappaport S., 2000, ApJ, 532, L47

Roberts M. S. E., 2013, in van Leeuwen J., ed., Proc. IAU Symp. 291, Neutron Stars and Pulsars: Challenges and Opportunities after 80 Years. Cambridge Univ. Press, Cambridge, p. 127

Robinson C., Lyne A. G., Manchester R. N., Bailes M., D’Amico N., Johnston S., 1995, MNRAS, 274, 547

Rohrmann R. D., Althaus L. G., Kepler S. O., 2011, MNRAS, 411, 781

Salaris M., Held E. V., Ortolani S., Gullieuszik M., Momany Y., 2007, A\&A, 476, 243

Serenelli A. M., Althaus L. G., Rohrmann R., Benvenuto O. G., 2001, MNRAS, 325, 607 
Serenelli A. M., Althaus L. G., Rohrmann R., Benvenuto O. G., 2002, MNRAS, 337, 1091

Sigurdsson S., Richer H. B., Hansen B. M., Stairs I. H., Thorsett S. E., 2003, Science, 301, 193

Sirianni M. et al., 2005, PASP, 117, 1049

Smarr L. L., Blandford R., 1976, ApJ, 207, 574

Tauris T. M., Savonije G. J., 1999, A\&A, 350, 928

Tauris T. M., van den Heuvel E. P. J., 2014, ApJ, 781, L13

Tauris T. M., Langer N., Kramer M., 2012, MNRAS, 425, 1601

Thygesen A. O. et al., 2014, A\&A, 572, A108 van Kerkwijk M. H., Bassa C. G., Jacoby B. A., Jonker P. G., 2005, in Rasio F. A., Stairs I. H., eds, ASP Conf. Ser. Vol. 328, Binary Radio Pulsars. Astron. Soc. Pac., San Francisco, p. 357

Verbunt F., Freire P. C. C., 2014, A\&A, 561, A11

Woodley K. A. et al., 2012, AJ, 143, 50

Zacharias N., Urban S. E., Zacharias M. I., Wycoff G. L., Hall D. M., Monet D. G., Rafferty T. J., 2004, AJ, 127, 3043

This paper has been typeset from a $\mathrm{T}_{\mathrm{E}} \mathrm{X} / \mathrm{LAT} \mathrm{T} \mathrm{X}$ file prepared by the author. 